\title{
Generating Realistic Images from Hydrothermal Plume Data
}

\author{
K. Santilli* \\ Rutgers University
}

\author{
K. Bemis ${ }^{\dagger}$ \\ Rutgers University
}

\author{
D. Silver ${ }^{\ddagger}$ \\ Rutgers University
}

\author{
J. Dastur ${ }^{\S}$ \\ Rutgers University
}

\author{
P. Rona** \\ Rutgers University
}

\begin{abstract}
Most data used in the study of seafloor hydrothermal plumes consists of sonar (acoustic) scans and sensor readings. Visual data captures only a portion of the sonar data range due to the prohibitive cost and physical infeasibility of taking sufficient lighting and video equipment to such extreme depths. However, visual images are available from research dives and from the recent IMAX movie, Volcanoes of the Deep Sea. In this application paper, we apply existing lighting models with forward scattering and light attenuation to the 3D sonar data in order to mimic the visual images available. These generated images are compared to existing visual images. This can help the geoscientists understand the relationship between these different data modalities and elucidate some of the mechanisms used to capture the data.
\end{abstract}

CR Catagories: I.3.7 [Computer Graphics]: Three-Dimensional Graphics and Realism-Raytracing; J.2 [Physical Sciences and Engineering]: Earth and atmospheric sciences

Keywords: Applications of volume graphics and volume visualization; Earth, Space, and Environmental Sciences Visualization; PC-based volume graphics; Volume Rendering

\section{INTRODUCTION AND Motivation}

Hydrothermal plumes form where hot fluids discharge from seafloor vents (commonly called black smokers) on mid-ocean spreading ridges. These hot buoyant fluids entrain the ambient seawater as they rise and are important to understanding the global transport of heat and chemicals in the ocean (which is a factor in climate studies) and to understanding the evolution of sea microbes.

Geologists, acoustical geophysicists and computational fluid dynamicists (CFD) are all involved in the study of seafloor hydrothermal plumes, and they all "see" plumes in different ways. The field geologists, in particular, are used to looking at optical photographs and videos. These are either taken directly of underwater hydrothermal plumes, where the limited visibility constrains the range and features, or are taken in the laboratory during a plume experiment. Geophysicists look at "acoustic" data, and acoustic images are significantly different from optical images. To optical tools, plumes have opaque cores, irregular but sometimes sharp edges, and slowly fading tops. Acoustic imaging sees all the way through the plume core to the other side but sees only gradational edges, because plumes are nearly transparent to transmission of acoustic waves although they do scatter them.

\footnotetext{
*email: kls93@caip.rutgers.edu

†email: bemis@rci.rutgers.edu

‡email: silver@caip.rutgers.edu

§email: jdastur@caip.rutgers.edu

**email: rona@imcs.rutgers.edu
}

Additionally, traditional 3D visualization is largely threshold based so the plume appears to have a sharp top (as an artifact of the chosen threshold). Computational fluid dynamicists see plumes as mechanisms of turbulent transport; they tend to focus on the size and speed of eddies rather than properties of the mean flow. Nevertheless, computationalists are more familiar with looking at isosurfaces within plumes than geologists are because CFD model results are 3D volumes just like sonar data and, hence, visualized with the same or similar tools.

The motivation for this study is to try to create more realistic plume images from the acoustic data to help bridge the gap between these fields and help the geologists understand the acoustical geophysicists data. Our goal is to use the particle concentration implicit in the acoustic backscatter intensity to recreate the optical image a camera would see given a particular lighting level.

An additional motivation for visualizing the acoustic data as optical data (i.e., converting acoustic images to optical images) is the greater understanding of the acoustic interaction with the plume and the properties of the plume. Our Vent Imaging and Processing (VIP) group is working to determine the relative roles of different backscatter mechanisms that produce acoustic images of black smoker plumes. We have assumed that Rayleigh backscatter (scattering by particles that are small relative to the wavelength of the acoustic pulses used to ensonify a plume) is the principal mechanism. Other possible backscatter mechanisms are density discontinuities related to turbulent structure and temperature variations within the buoyant plumes. By using visualization techniques, specifically realistic lighting models [7][8][19][25], we can convert the plume image from mechanical waves (sound) to electromagnetic waves (light). The acoustic data are the observed volume backscattering coefficients for Rayleigh scattering and the resulting optical images will model the Mie scattering of visible light. This will, in conjunction with other tests, help us to sort out backscattering mechanisms and their relative importance.

In the next section, we describe how sonar images of plumes are acquired. This is followed by a description of the realistic rendering techniques most relevant to this study. Results of applying the realistic rendering to the acoustical images are described in Section 3. These computed images are compared to "real" images (Figure 1) taken from an underwater cruise [22][21]. Because the lighting techniques are general, different lighting conditions can be simulated by changing the parameters of the model. In Section 4, we apply an IMAX filming based lighting model to the acoustic data and compare these results to images from a recent IMAX film, Volcanoes of the Deep Sea [26]. This film captured spectacular images of hydrothermal plume from a site in the Pacific and several sites in the Atlantic; the images shown in Figure 1 are from the RIDGE 2000 Integrated Study Site at $9^{\circ} \mathrm{N}$ on the East Pacific Rise (EPR) and the vent complex called Snakepit at $23^{\circ} \mathrm{N}$ on the Mid-Atlantic Ridge. Note the different color of the plumes, which is both a function of the materials present in the plume and the distance of the camera and lights. 

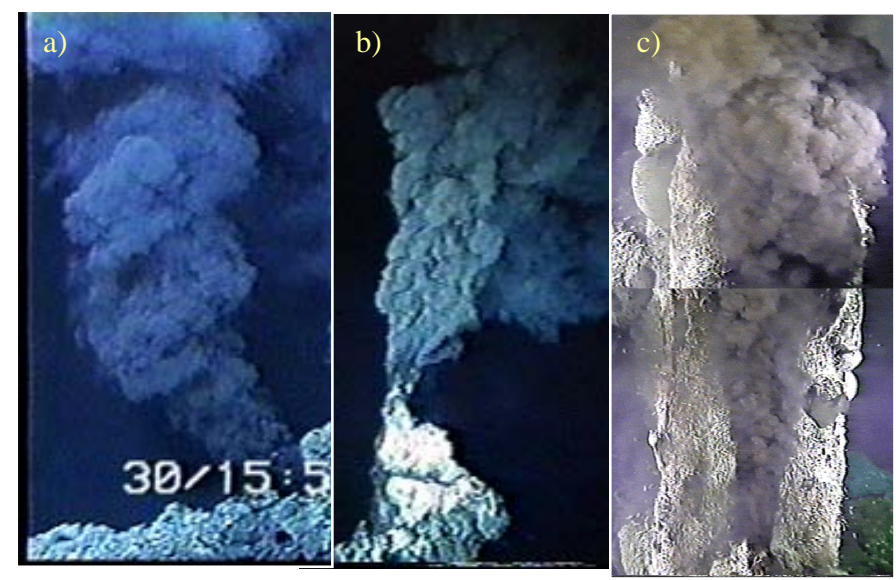

Figure 1: (a) The first image is a cropped video still from the VIP 2000 cruise in July 2000 of a black smoker on Grotto Vent in the Main Endeavour Field on the Juan de Fuca Ridge. The image is approximately $0.5 \mathrm{~m}$ wide. (b-c) The other two images are cropped video stills from the IMAX movie Volcanoes of the Deep Sea, produced by The Stephen Low Company and Rutgers University [26]. These images are from video version of the film. (b) A vent at $9^{\circ} \mathrm{N}$ on the East Pacific Rise. Image is approximately $2 \mathrm{~m}$ wide. (c) This plume at Snakepit on the MidAtlantic Ridge (MAR) rises in front of a shrimp coated tower. This figure is a mosaic of 3 images, which is approximately $2 \mathrm{~m}$ wide.

\section{BACKGROUND}

Sub-seafloor hydrothermal convection systems discharge as plumes from point sources and as seepage from areas of the ocean bottom. The plumes originate as clear, hot (c. $150^{\circ}$ to $400^{\circ} \mathrm{C}$ ) solutions that vent from mineralized chimneys, precipitate dissolved metals as particles to form black or white smokers as they turbulently mix with ambient seawater, and buoyantly rise hundreds of meters to a level of neutral density where they spread laterally. Hydrothermal flow regimes are being intensively studied for their role as quantitatively significant agents of dispersal of heat, chemicals, and biological material into the ocean from subseafloor hydrothermal convection systems on a global scale (e.g., [9]).

Table 1: Minerals found in plume particles

\begin{tabular}{lllll} 
Mineral & Formula & $\begin{array}{l}\text { Reflected } \\
\text { Color }\end{array}$ & Albedo[2] & $\begin{array}{l}\text { Size[10] } \\
(\mu \mathrm{m})\end{array}$ \\
\hline Anhydrite & $\mathrm{CaSO}_{4}$ & white & 0.0085 & $10-500$ \\
Chalcopyrite & $\mathrm{CuFeS}_{2}$ & yellow & $0.12-0.41$ & $10-60$ \\
Pyrite & $\mathrm{FeS}_{2}$ & cream & $0.38-0.57$ & $10-60$ \\
Pyrrhotite & $\mathrm{Fe}_{(1-\mathrm{x})} \mathrm{S}$ & pink beige & $0.28-0.44$ & $0.1-5$ \\
Sphalerite & $(\mathrm{Zn}, \mathrm{Fe}) \mathrm{S}$ & grey & $0.16-0.19$ & $50-100$
\end{tabular}

The particles in hydrothermal plumes depend on the chemistry and temperature of the fluids exiting the seafloor. High temperature $\left(250-350^{\circ} \mathrm{C}\right)$ fluids from black smokers (Figure 1) are typically characterized by the precipitation of metal-rich sulfide and sulfate minerals (chalcopyrite, pyrrhotite, pyrite, sphalerite) while lower temperature $\left(70-300^{\circ} \mathrm{C}\right)$ fluids from white smokers typically precipitate amorphous silica, pyrite and barite (Feely et al., 1987). The exact mineral assemblage in black smoker plumes varies between regions. Precipitates in plumes along the Endeavour Segment of the Juan de Fuca Ridge (e.g., Grotto Vent in Figure 1a) are dominated by chalcopyrite and anhydrite [10].

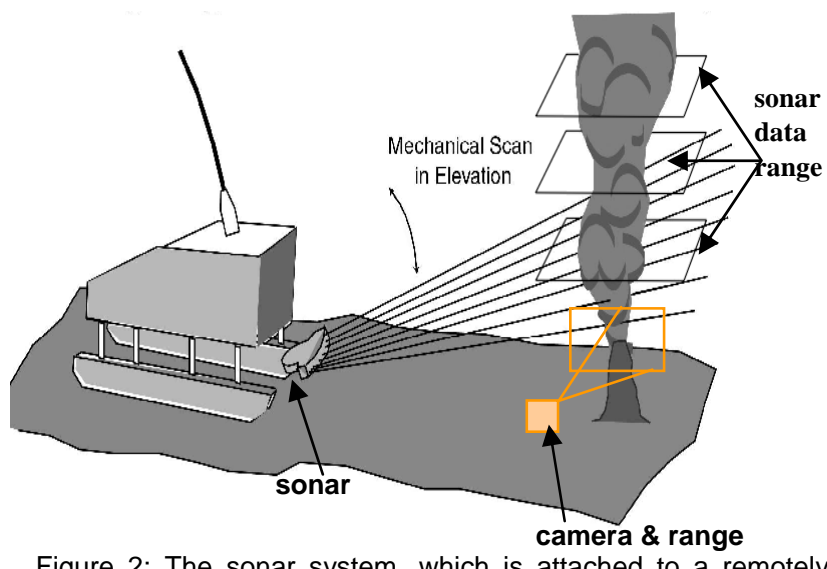

Figure 2: The sonar system, which is attached to a remotely operated vehicle, scans a hydrothermal plume. Three volume slices are shown. The video images in Figure 1 are taken when the vehicle moves in closer (orange box marks the camera position). Note the different range for the sonar and camera.

Pyrrhotite is more important in some plumes along the East Pacific Rise [10]. Particle composition also varies vertically in the plume [10] [23]. Table 1 gives the chemical formula and some optical properties for the minerals most likely to be found in our study area (Endeavour).

\subsection{Acoustic Remote Sensing}

Our acoustic images of plumes are based on the intensity of Rayleigh backscatter from metallic mineral particles (sulfides, oxyhydroxides, sulfates) precipitated from high-temperature hydrothermal solutions and suspended as "smoke" in the plumes [17]. The particles are small (microns) relative to the wavelength of the acoustic frequencies used $(\sim 1 \mathrm{~cm}$ at 200 to $330 \mathrm{kHz})$. At sufficiently low particle concentrations (for no multiple scattering) backscatter intensity is directly proportional to concentration of suspended particles as the product of total particle load and a factor dependent upon average particle mechanical properties (density, bulk modulus, and radius).

The volume backscattering coefficient, which is easily and accurately computed from the raw acoustic data, plays a similar role for sound as the extinction coefficient does for light. However, Rayleigh backscattering and Mie forward scattering have different dependencies on wavelength (or wavenumber $\mathrm{k}$ ) and particle size (radius $r$ ). In the case of Mie scattering (for both light and sound where the wavelength is much smaller than the particle), the extinction cross-section is approximately twice the actual particle's cross-sectional area [11][12] (see Table 2 for terms):

$$
\sigma_{\text {Mie }}=2 \pi r^{2} \text {, when } k r>>1
$$

In the case of Rayleigh scattering (wavelengths much larger than the particles), the backscattering cross-section depends on the fourth power of the ratio between particle size and wavelength [12]:

$$
\sigma_{\text {Rayleigh }}=r^{2} \frac{25(\mathrm{kr})^{4}}{36}, \text { when } k r<<1
$$

In this study, the sonar is operating at a frequency of $200 \mathrm{kHz}$ and the particles are approximately $500 \mu \mathrm{m}$. The extinction scale factor $\lambda$, which is the ratio of the Mie extinction cross-section and the Rayleigh backscattering cross-section, used in the opacity 
transfer function (described in Section 3.1.2) is estimated as $1.79 \times 10^{4}-5.72 \times 10^{5}$ based on the wavelength of the sonar's transmitted pulse $(0.75 \mathrm{~cm})$ and a range of particle sizes of 500 $1000 \mu \mathrm{m}$ (very limited data is available and the methodologies of particle size measurement are suspect).

\subsection{Sonar Image Acquisition}

The sonar data used in this study were obtained as part of the Vent Imaging Pacific 2000 (VIP 2000) cruise during which several types of sonar observations were made within the Main Endeavour Field on the Juan de Fuca Ridge (Figure 3). In this recent experiment [22][21], a sonar system (Kongberg Simrad Mesotech SM 2000) operating at 200 $\mathrm{kHz}$ and mounted on the tethered remotely operated

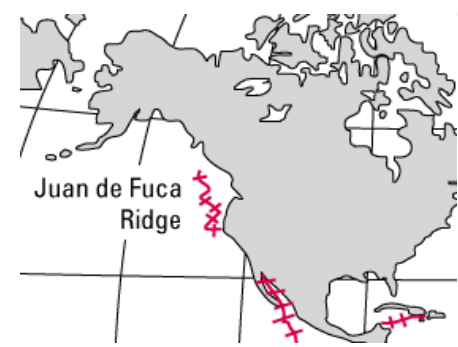

Figure 3. The July 2000 experiment was conducted on the Endeavour segment of the Juan de Fuca Ridge. vehicle ROV JASON, operated by the Deep Submergence Group of the Woods Hole Oceanographic Institution, was used to acquire acoustic datasets. Figure 4 shows three-dimensional images of plume structure using direct visualization techniques [3][20]. The 3D images are formed by means of a combination of time gating (for resolution in range), digital beam forming (for resolution in azimuth), and mechanical scanning (for resolution in elevation). Range resolution is between $0.2 \mathrm{~m}$ and $2.0 \mathrm{~m}$ depending upon the transmitted waveform and the width of the window used for averaging in the sonar time series. Azimuthal and elevation resolution are both equal to the sonar beamwidth $\left(1.4^{\circ}\right.$ between half-maximum-power points). The system scans a plume in 2 minutes and is calibrated to record absolute backscatter intensity.

The sonar data were acquired with ROV JASON stationary on the West wall of the Endeavour segment near the Grotto vent complex (Figure 2). Video observations show that the Grotto plume is produced by coalescence of at least five vents within a 3 $\mathrm{m} \times 3 \mathrm{~m}$ area. The several smaller plumes issuing near the summit of Grotto are estimated to have coalesced at a height of about 4-5 $\mathrm{m}$. A significantly lower height of coalescence would have been observable in the video imagery and a significantly higher height would have allowed resolution of separate plumes by the sonar. Owing to sidelobe interference by the Grotto edifice, the sonar could not image plume structure for heights less than $5 \mathrm{~m}$. It is important to note that the acoustic images and the camera stills (Figure 1) come from different regions of the plume.

The results of conventional visualization methods, such as isosurfaces and basic raytracing, tend to have edges that are too solid and omit entirely any less intense peripheral areas that indicate which way the plume is drifting or how the particles are dispersing as the plume mixes with the surrounding water. Examples of this are shown in Figure 4. While these images are understandable to most visualization scientists, geologists do not tend to think in thresholds and prefer to see images that resemble photographs.

\subsection{Realistic Lighting Models}

Methods for realistically rendering of translucent objects, have been, and continue to be, a popular focus for research in the scientific visualization community [11]. The lighting model that most significantly influenced our implementation was the particle- sensitive scattering model proposed in Riley et al. [19]. In that model, Riley et al. expands upon the scattering model introduced in Nishita et al. [15] by allowing the optical properties such as the extinction coefficients and phase function for scattering to vary through the dataset. It also incorporates the approximations for highly dominant forward scattering described in Kniss et al. [14], but without the chromatic absorption term from that work.

Most of the work listed above deals with rendering realistic images of clouds. Realistic plume visualization is in many ways parallel to the realistic visualization of clouds and weather data. Like clouds, plumes are made up of small, suspended particles and their particle densities can vary greatly within the structure. Also, while most plumes have a much lower albedo than clouds, their appearance is still significantly affected by the strong forward scattering effects [5][19]. However, water as a medium has non-negligible opacity and strongly chromatic attenuation.

\section{Plume Application}

Although the sonar data only provides a measurement of the volume scattering coefficient throughout the volume, this result can be correlated with the particle density (or particle concentration to a geologist) [16]. By applying the known properties of seawater, suspended particles, and the minerals found in plumes, the particle density can be used to calculate reasonable values for the volume's various optical properties. For convenience and ease of calculation, we then perform the rendering in two phases (similar to [8][7]), the first of which is a preprocessing step in which the light propagation through the volume is calculated for each individual light source. The second phase is a direct volume rendering process in which we can incorporate the light contributions from any combination of the preprocessed lights and vary the viewing angle.
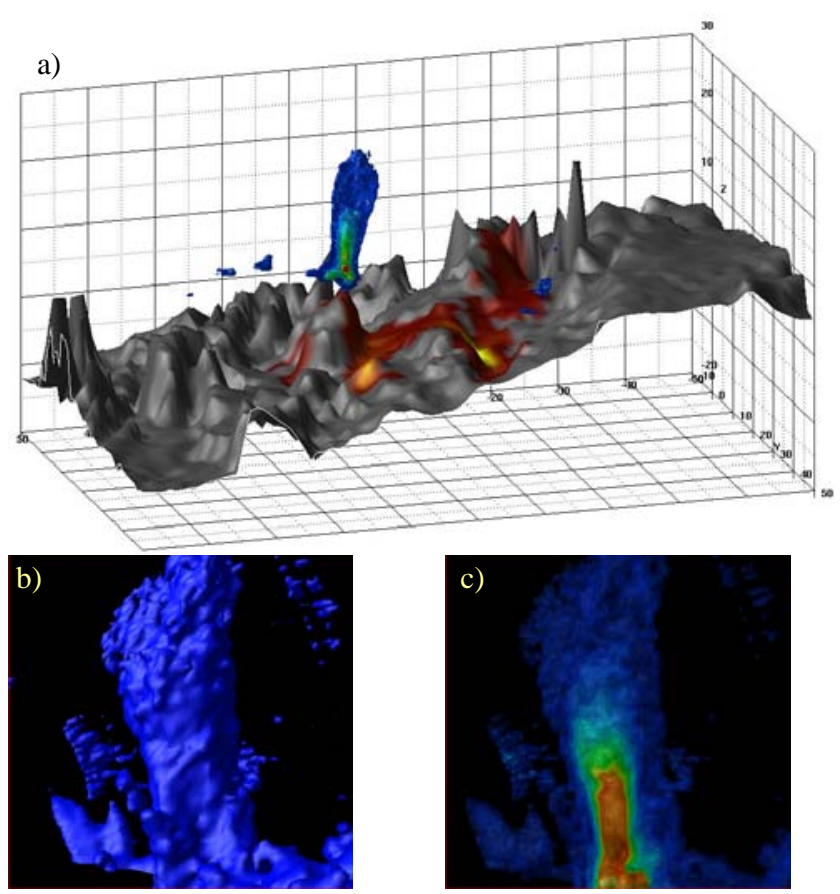

Figure 4: (a) A solid contour rendering of the acoustic data used in this study is shown above the actual bathymetry of the site (Grotto Vent in the Main Endeavour Field on the Endeavour Segment of the Juan de Fuca Ridge). (b) A simple isosurface of acoustic data. (c) A solid contour rendering of the same dataset. 
Table 2: Variables list

\begin{tabular}{cc} 
Variable & Definition \\
\hline $\mathrm{L}(\vec{w})$ & Intensity at location $\vec{w}$ \\
$\mathrm{~T}(\vec{s}, \vec{w})$ & Total attenuation between $\vec{s}$ and $\vec{w}$ \\
$\mathrm{~L}_{\mathrm{bg}}$ & Background light intensity \\
$\mathrm{C}(\overrightarrow{\mathrm{s}})$ & Reflective color at $\vec{s}$ \\
$\mathrm{P}(\phi)$ & Scattering phase function, function of angle $\phi$ \\
$\mathrm{L}_{\mathrm{l}}()$, & Total incident light at s from direction W \\
$\mathrm{L}_{i}$ & Intensity of light $i$ \\
$\delta(\phi)$ & A displacement that gives a direction at an angle $\mathrm{f}$ \\
$\tau(\vec{s})$ & Coefficient of extinction by occlusion at s \\
$\mathrm{f}(\vec{s})$ & Sonar data value at s \\
$\sigma$ & Cross-section \\
$\mathrm{k}$ & Wave number (ratio of $2 \pi$ and wavelength) \\
$\mathrm{r}$ & Particle radius \\
$\mathrm{kr}$ & Product of wave number and particle radius
\end{tabular}

\subsection{Lighting Model}

\subsubsection{Calculation of Optical Properties}

Calculation of the local light contribution (see Section 3.1.2) depends on the sonar data and the optical properties of seawater and the particles in the plume. These properties include scattering phase functions, reflective color, chromatic absorption, and opacity. A list of the variables used here is provided in Table 2, and a list of constants and their values is provided in Table 3.

The scattering phase function determines what percentage of the colliding light will scatter and in what direction. Since at least $90 \%$ of scattering from particles with a radius much larger than the wavelength of the incident light (500 $\mu \mathrm{m}$ compared with 0.5 $\mu \mathrm{m}$ ) occurs within $5^{\circ}$ arc, we approximate the scattering phase as two cumulative constants, described in 3.1.2 [5][19].

Reflective color and chromatic absorption determine the color of the final image. The reflective color used for the plume particles is based on data available from reference resources (e.g., [2]) on the reflective color of minerals identified as suspended particles in the plume. Chromatic absorption accounts for the wavelength dependence of light attenuation in water. The extinction constants used here are taken from [18], using the values at wavelengths of $680 \mathrm{~nm}, 540 \mathrm{~nm}$, and $470 \mathrm{~nm}$ for red, green, and blue, respectively. Most of the available data of this type is based on pure water, but the properties of seawater are shown to be very similar [27]. Chromatic extinction in the medium can be calculated with a standard extinction equation for each color channel, as discussed in [14].

Opacity is also calculated with a standard extinction equation [11]. The sonar data provide information about the scattering cross-section of the particles in the plume under study. This feature is strongly related to the extinction, as described in [19].

\subsubsection{Light Propagation}

The lighting and rendering model is similar to that presented in

Table 3: Constants list

\begin{tabular}{cccc} 
Symbol & Value & Definition \\
\hline$\lambda$ & 17900 & Extinction scale factor \\
\hline & R & 0.465 & \\
$\eta$ & G & 0.0474 & Coefficient of extinction due \\
& B & 0.0106 & to absorption into the medium
\end{tabular}

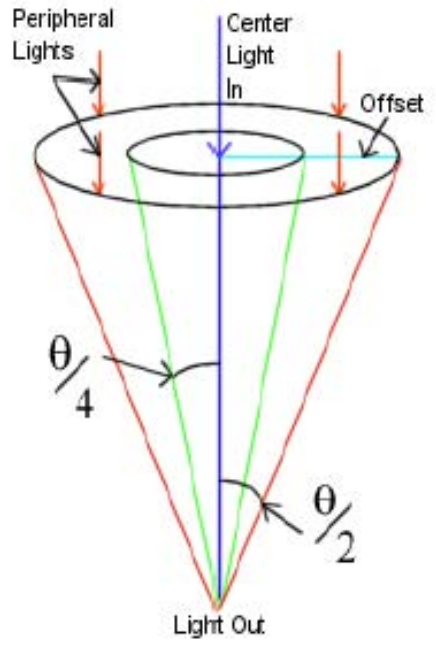

Figure 5: An illustration of the sampling locations and the role of the major scattering angle, $\theta[19]$.

[19] and summarized by the equation for the intensity at each pixel location:

$$
\begin{aligned}
\mathrm{L}(\vec{w})= & \mathrm{T}(0, \vec{w}) \mathrm{L}_{\mathrm{bg}}+ \\
& \int_{0}^{\vec{w}} \mathrm{~T}(\vec{s}, \vec{w}) \mathrm{C}(\vec{s})\left[\int_{4 \pi} \mathrm{P}(\psi(\Omega)) \mathrm{L}_{1}(\vec{s}, \Omega) \mathrm{d} \Omega\right] \mathrm{d} \vec{s}
\end{aligned}
$$

$\mathrm{T}(\vec{s}, \vec{w})$ is the total attenuation between points $\vec{s}$ and $\vec{w}$. $\mathrm{L}_{\mathrm{bg}}$ is the background light intensity. $\mathrm{C}(\vec{s})$ is the reflective color at point $\vec{s} . \mathrm{P}(\psi(\Omega))$ is the scattering phase function as a function of the angle between the incident light and the viewpoint, $\psi(\Omega) . \mathrm{L}_{1}(\vec{s}, \Omega)$ is the total incident light at point $\vec{s}$ in the direction $\Omega$. In the light preprocessing phase, each light's contribution to $\mathrm{L}_{1}(\vec{s}, \Omega)$ is calculated.

For each light $i$, the intensity at point $\vec{s}$ is given by equation (4) [19]:

$$
\begin{aligned}
& \mathrm{L}_{\mathrm{l}, i}\left(\vec{s}, \omega_{i, \vec{s}}\right)=\mathrm{L}_{i} \mathrm{~T}\left(\vec{s}, \overrightarrow{l_{i}}\right)+ \\
& \int_{\vec{l}_{i}}^{\vec{s}} \mathrm{~T}\left(\vec{s}^{\prime}, \vec{l}_{i}\right)\left[\int_{\theta} \mathrm{P}(\phi) \mathrm{L}_{1, i}\left(\vec{s}^{\prime}, \omega_{i, \vec{s}^{\prime}}+\delta(\phi)\right) \mathrm{d} \phi\right] \mathrm{d} \vec{s}^{\prime}
\end{aligned}
$$

Here, $\vec{l}_{i}$ is the position of light $i$, and $\omega_{i \vec{s}}$ is the direction from $\vec{s}$ to $\vec{l}_{i} . L_{i}$ is the intensity of light $i$. The first term represents the unimpeded light that reaches $\vec{s}$ and the second term incorporates the forward scattering component. The inner integral is evaluated over the over the arc angle $\theta . \delta(\phi)$ is a displacement that gives a direction at an angle of $\phi$ from $\omega_{i, \vec{s}^{\prime}}$.

This forward scattering effect is implemented using the conical sampling region and integrated phase function described in [19]. A cone, as shown in Figure 5, is projected from the sampling point towards the light source. A single central sample and several peripheral samples of the light intensity are taken. The central sample is used to calculate the intensity contributions from direct light and light that was forward scattered within an angle of $\theta / 4$. The peripheral samples are averaged and the result is used to estimate the intensity contribution from light that scattered at an angle between $\theta / 4$ and $\theta / 2$. Since the scattering percentage is being calculated for regions instead of single points, the scattering phase function is integrated over each of the two regions and the resulting constants are used in the implementation. For this application, eight peripheral samples were used instead of four, 


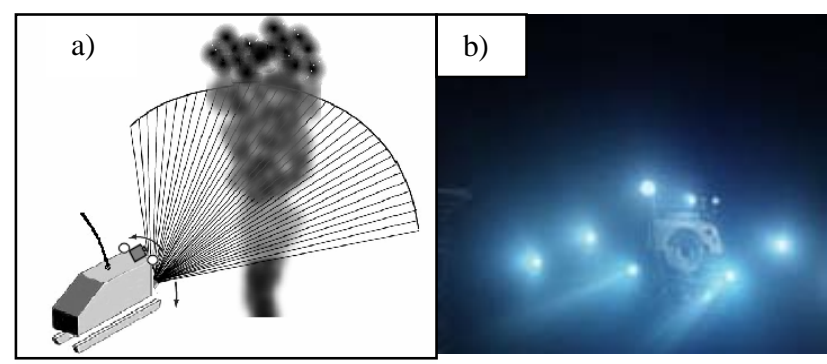

Figure 6: The lighting setups for the two cases discussed in the text are quite different. (a) The VIP 2000 cruise video used two lights mounted near the camera on the front of the ROV JASON (400 W). (b) The IMAX filming used up to 8 lights spaced out on a set of booms around DV ALVIN with a total of $4400 \mathrm{~W}$ [26]. Filming was done from both inside DV ALVIN and a separate unit outside.

and the phase function is a Henyey-Greenstein function with a forward-scattering bias of 0.85 , i.e:

$$
\mathrm{P}(\phi)=\frac{1}{4 \pi} \frac{1-0.85^{2}}{\left(1+0.85^{2}-2 \cdot 0.85 \cos \phi\right)}
$$

In addition to the forward scattering effect, a chromatic extinction term has been added to $\mathrm{T}(\vec{s}, \vec{w})$ to account for the strongly chromatic absorption of light traveling through water. The resulting equation is presented in equation (6) [11][14]:

$$
\begin{gathered}
\mathrm{T}(\vec{s}, \vec{w})=\exp \left(-\int_{\vec{w}}^{\vec{s}}\left(\tau\left(\vec{s}^{\prime}\right)+\eta\right) \mathrm{d} \vec{s}^{\prime}\right) \\
\tau(\vec{s})=\lambda \mathrm{f}(\vec{s})
\end{gathered}
$$

where $\tau(\vec{s})$ is the usual extinction coefficient due to occlusion and $\eta$ is the (RGB dependent) coefficient for extinction due to absorption by the medium. The origin of the scale factor of $\lambda$ in $\tau(\vec{s})$ is described in Section 2.1. The RGB values of $\eta$, mentioned in Section 3.1.1, are listed in Table 3.

\section{Results}

\subsection{Cruise Conditions}

Figure 9a displays the result of applying the cruise lighting model to our acoustic dataset. The acoustic data was taken at the Grotto Vent Site in the Main Endeavour Field on the Juan de Fuca Ridge. Its resolution is $0.5 \mathrm{~m} \mathrm{x} 0.5 \mathrm{~m} \mathrm{x} 0.5 \mathrm{~m}$, and this dataset covers a region that is $100 \mathrm{~m} \times 60 \mathrm{~m} \times 55 \mathrm{~m}$ (other acoustic data may cover

\begin{tabular}{|c|c|c|c|}
\hline Render Model & VIP 2000 Cruise & IMAX 3 & IMAX 5 \\
\hline Number of lights & 2 & 3 & 5 \\
\hline Positions*: & & & \\
\hline Light 1 & $(-2,0,0)$ & $(-10,-1,0)$ & $(-2,0,0)$ \\
\hline Light 2 & $(2,0,0)$ & $(10,-1,0)$ & $(2,0,0)$ \\
\hline Light 3 & & $(0,5,0)$ & $(-10,-1,0)$ \\
\hline Light 4 & & & $(10,-1,0)$ \\
\hline Light 5 & & & $(0,5,0)$ \\
\hline Base Attenuation & $\begin{array}{c}(0.92,0.97 \\
0.9925)\end{array}$ & $\begin{array}{c}(0.97,0.985 \\
0.9925)\end{array}$ & $\begin{array}{c}(0.97,0.985 \\
0.9925)\end{array}$ \\
\hline
\end{tabular}
a different range). An actual photographic image taken during this

Table 4. Lighting setups used for renderings

*Positions given relative to camera's initial position. cruise is shown in Figure 1. The photograph has a smaller field of view than the acoustic image, but appears to have a similar one due to the inherent self-similarity of plumes. The photograph shows an approximately $0.5 \mathrm{~m}$ field of view; the acoustic data set is $60 \mathrm{~m}$ wide but starts $\sim 3 \mathrm{~m}$ below the vent. The camera and lighting position during the cruise is shown in Figure 6a and are at approximately the same position in front of the plume. The parameters for this scenario are shown in Table 4. The image in Figure $9 \mathrm{a}$ is quite dark because the cruise lighting is very low and the modeled camera is at the sonar position ( $20 \mathrm{~m}$ from plume) rather than the camera position of the video stills $(\sim 5 \mathrm{~m}$ from plume).

\subsection{IMAX Conditions}

The recent IMAX film, Volcanoes of the Deep Sea [26], filmed some magnificent images of hydrothermal vents in both the Pacific and Atlantic oceans. Stills from the movie are shown in Figure $1 \mathrm{~b}-\mathrm{c}$ above; these particular images are of a vent at $9^{\circ} \mathrm{N}$ EPR in the Pacific Ocean and of a vent at Snakepit on the MAR in the Atlantic Ocean. The IMAX lighting setup can be seen in Figure 6b. An array of 3-8 lights shine directly on the plume. During filming different submersibles were used to capture different lighting and camera positions. Figure $1 \mathrm{~b}$ is an image with front lighting.

In Figure 7b, an acoustic data set is rendered with IMAX 3 lighting conditions including a big spotlight, whiter light, and more total lights (note, the acoustic data is from a different site then the IMAX film). Notice how much more brightly the plume is illuminated in Figure 7b compared to Figure 9a, although very little more of the plume is visible. The billowing effect of eddies is also more noticeable in Figure 7b than in Figure 9a. The distinct identity of the plume from the surrounding water is apparent in the lighting rendering of Figure $7 \mathrm{~b}$ but not in the standard rendering in Figure 7a.

Figure 10 shows a lighting panorama (a sequence of images forming a panorama) created by positioning the spotlight at five locations along a 90 degree arc around the front of the plume. The lighting position is static. Note how this type of image would be very difficult to take underwater, as two different vehicles would be necessary (one for the light, one for the camera). An animation showing this effect can be seen at [24].

\section{Discussion AND CoNCLUSION}

Part of the motivation for reproducing the acoustic data as lighting panoramas is that the video images of plumes cover a very small area; even the better cameras and the stronger lights of the IMAX setup produced only $2 \mathrm{~m}$ by $6 \mathrm{~m}$ images (see Figure 1 ). The much larger dimensions of the acoustic data $(100 \mathrm{~m} \times 60 \mathrm{~m} \times 55 \mathrm{~m})$ offer the potential to see so much more of the plume. However, the standard volume rendering techniques are threshold based producing isosurfaces or ray-tracings with color and opacity as direct thresholds. The lighting techniques describe in Section 3 allow use of a more sophisticated transfer function and inclusion of the effects of shadowing and scattering.

Comparison of the generated images and the video images must take into account the fact that the plume gets thinner and more wispy as it rises (this is starting to occur at the top of the IMAX images in Figure 1) because of the dilution of the particle density with entrainment of seawater. Otherwise, the plume structure is self-similar in that the size relation of the eddies to the diameter of the plume is constant over most of the $100-300 \mathrm{~m}$ of rise in the buoyant plume and the dynamic processes of turbulence and entrainment are relatively constant over the buoyant rise. That said, the rest of this section compares the video and generated 

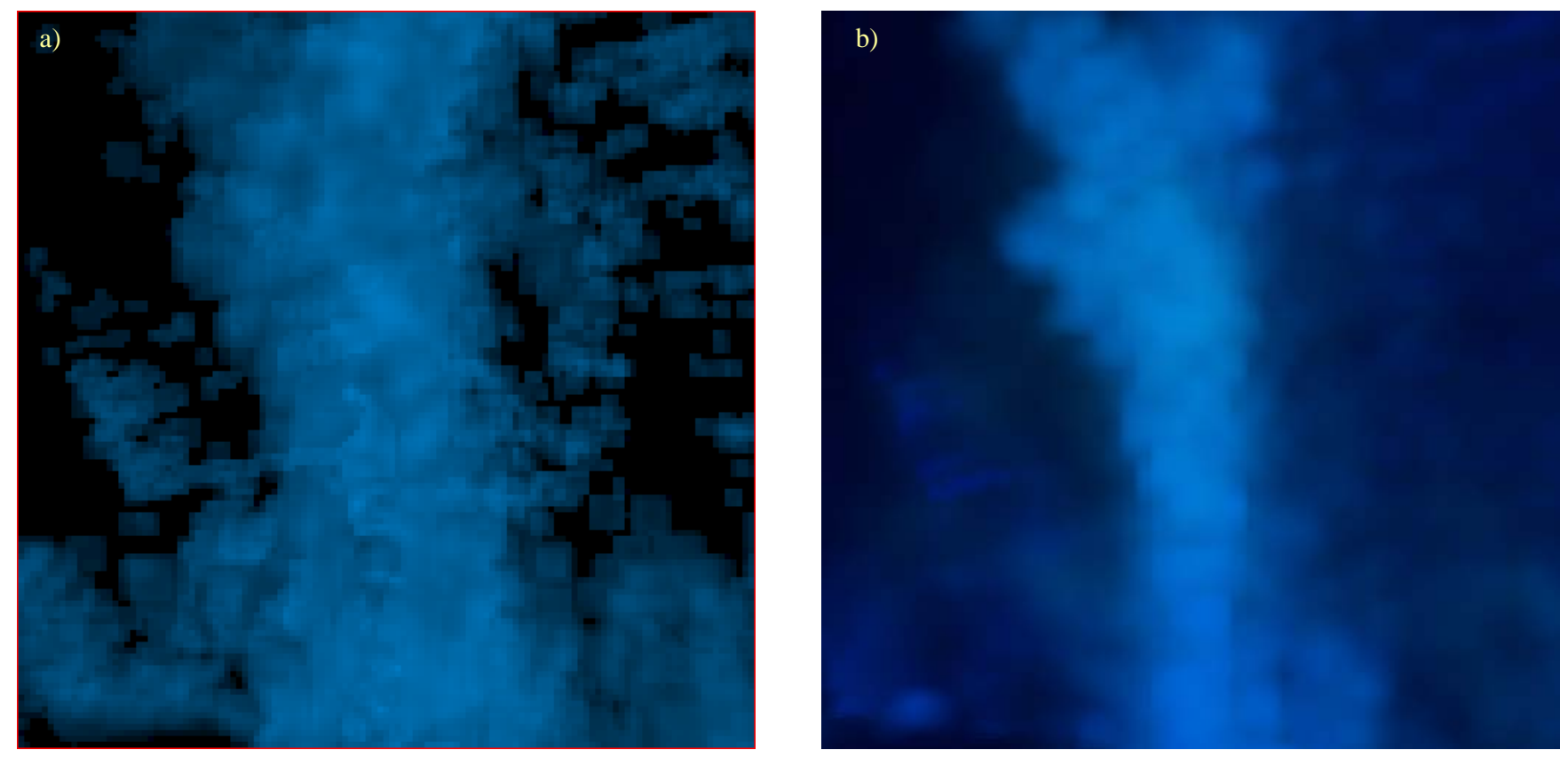

Figure 7. (a) Basic raytracing of the subset of acoustic data used in this study. The subset has physical dimensions of $60 \mathrm{~m}$ by $60 \mathrm{~m}$ by $45 \mathrm{~m}$. Many of the blue areas outside the central plume are noise or artifacts of sonar sidelobes encountering the rocky seafloor. (b) Image of the acoustic data in Figure 4 rendered using IMAX 5 lighting conditions (see Table 3). Notice that the plume now has a distinct identity from the surrounding water. The eddy shapes are also somewhat more apparent.

images, discusses their differences and suggests techniques for future improvements.

Figure 9 shows three images that compare the effect of changing the lighting setup (see Table 4) while the extinction factor, camera location, and albedo are held constant. The generated images using the cruise lighting setup have a similar blue color to the cruise video images. The blue color is due to the absorption of red, and to a lesser extent green light, by the water. The generated image shows less detailed eddies, although it does show some indications of eddy shape and size. The lack of fine detail in the cruise-setup images is due to the coarse resolution of the acoustic data - it is difficult to produce $\mathrm{cm}$ scale features with $\mathrm{m}$ scale data. The plume in the cruise-setup images also appears less opaque. This may just be a result of the increased dilution of the plume with height (and concurrently expanding width). As stated in Section 4.1, the generated image is very dark which is consistent with our inability to see the plume during acoustic data acquisition. This is part of the utility in generating images from the acoustic data: the plume becomes more visible as more lights are added (see Figure 9b-c). We anticipate further exploitation of this effect to show geologists what the plumes would look like if they could always have IMAX like lighting (which is prohibitive because of the high power requirements and cost of the lights).

The generated images using the IMAX 3 and IMAX 5 lighting setups are brighter (and even brighter) with slightly less blue tones than the cruise-setup images corresponding to the slightly reduced blue tones in the IMAX video still (Figure 1b) compared to the cruise video image (Figure 1a). The lack of blue in the second IMAX still (Figure 1c) relates to the close proximity of the camera to the plume; the scope is the same because several stills were combined in a mosaic.

The IMAX-setup image shows more detailed eddies than the cruise-setup image, although not as much detail as the video images. The lack of fine detail in the generated images is due to the coarse resolution of the acoustic data. The apparent increase in detail between the cruise and IMAX setups is likely just better illumination, which clarifies the details that are there. The plume in the IMAX-setup images appears more opaque than in the cruise-setup images, which is probably related to the greater amount of light reflected back; there was no change in the actual opacities. Figure 10 shows a panoramic sequence of views of the plume with the camera moving from $45^{\circ}$ right of the light source to $45^{\circ}$ left of the light source and also an image from behind the plume. The lighting panorama shows the three-dimensional structure of the plume and its eddies. The opacity of the plume core is very clear and slight bending of the plume due to currents can also be seen (especially at later times [24]).

Figure 11 shows a sequence of generated images using different values of the extinction scale factor (see Sections 2.1 and 3.1.2). Because there are few samples of particles from the buoyant stem of hydrothermal plumes, we can only estimate the value of the extinction scale factor, which depends on particle size (because the Rayleigh scattering cross-section is a function of particle size). This series of images (Figure 11) shows that the brightness and apparent width of the plume in the generated images increases as the extinction scale factor increases and assumed particle size decreases. The change in the apparent width of the plume results from the background and edges of the plume becoming more visible as increasing the extinction scale factor shifts the opacity transfer function towards lower data values. The background values in the acoustic data reflect noise in the sonar system.

Comparison of the generated images with the cruise and IMAX video stills suggests that a particle size of $1000 \mu \mathrm{m}$ leads to the closest match with the generated images. This is larger than the particle sizes observed $(10-500 \mu \mathrm{m})$ but the particle sizes were obtained by using a vacumm pump to filter water samples through a fine mesh $(<10 \mu \mathrm{m}$ openings), which is known to disturb clumps (aggregations) of particles. In future at sea experiments, we plan to use modern instruments based on lasers to determine the actual particle size distributions.

Figure 12 shows a sequence of images, which vary the albedo (reflectivity) assigned to the average particle modeled - Table 1 shows that the true albedo is a function of the particle chemical composition. Thus the differences in the series of generated 
images reflect our assumptions about the average particle composition. As stated in Section 2, the plumes on the Endeavour Segment (where Grotto Vent imaged here is located) are typically dominated by chalcopyrite and anhydrite [10], which have a low albedo $(<0.3)$. However, the generated image most consistent with the video stills suggests that the Grotto Vent plume may actually be dominated by pyrite, which has the highest albedo ( 0.5); this is chemically plausible for high temperature vents $[10]$.

Future improvements include exploring the parameters that effect opacity and color and adding additional scattering mechanisms. We have used the best possible choices for transfer functions from acoustic backscatter to extinction coefficient based on available data (see Section 3.1), but have not conducted a systematic survey of the effects of varying the parameters (sensitivity analysis). We intend to explore the controls on the apparent opacity and color of the plume by changing the reflective color and opacity properties of the particles in conjunction with the multiple hypotheses in the literature concerning particle composition [10][17][1][23][4]. We would also like to add the effects of other scattering mechanisms and sub-eddy-scale details based on turbulence models [14]. We plan to explore other existing acoustic data (including some closer to the EPR IMAX video) with this methodology.

This study shows that lighting models can be used to generate realistic optical images from acoustic data. This will improve understanding of seafloor hydrothermal plumes by clarifying the geologic interpretation of the acoustic data. In addition, changing the number of lights can be used to mimic the effect of different lighting conditions (Figure 11). Thus, the lighting model is effective and our future studies varying optical properties will address the relative importance of different types of particles in the plume's appearance.

\section{Acknowledgements}

The giant screen film, Volcanoes of the Deep Sea, is a production of the Stephen Low Company and Rutgers University (http://www.volcanoesofthedeepsea.com). The images shown here are from a video version of the original footage. We thank Drew Reed for his assistance in extracting high resolution images from the VIP2000 video coverage and the IMAX video. This material is based on work supported the National Science Foundation under Grant No. ACI-0312272.

\section{REFERENCES}

[1] E. Baker and G. Massoth, "Characteristics of hydrothermal plumes from two vent fields on the Juan de Fuca Ridge, northeast Pacific Ocean”, Earth and Planetary Science Letters, 85 (1-3), 1987, pp. 5973.

[2] D. Barthelmy, "Mineralogy Database”, http://www.webmineral.com.

[3] K. Bemis, D. Silver, P.A. Rona, and C. Feng, "Case study: a methodology for plume visualization with application to real-time acquisition and navigation”, IEEE Visualization 2000 Proceedings, 2000, pp. 481-494.

[4] M. Bertram, J. Cowen, R. Thomson, and R. Feely, "Compositional variability in the ascending fluxes from a hydrothermal plume", Journal of Geophysical Research, 107 (C11), 3191, doi:10.1029/2000JC000223, 2002.

[5] C. F. Bohren and D. R. Huffman, Absorption and Scattering of Light by Small Particles. John Wiley and Sons, 1983.

[6] W. de Leeuw, F. Post, and R. Vaatstra, "Visualization of turbulent flow by spot noise", Virtual Environments and Scientific Visualization '96, Proceedings of the Eurographics Workshops, M. Göbel, J. David, P. Slavik, and J. van Wijk, eds., Springer-Verlag, Wien, 1996.
[7] Y. Dobashi, T. Yamamoto, and T. Nishita, "Interactive rendering of atmospheric scattering effects using graphics hardware", Proceedings of the ACM SIGGRAPH/EUROGRAPHICS conference on Graphics hardware, 2002, pp. 99-108, 159.

[8] M. J. Harris and A. Lastra, Anselmo. "Real-time cloud rendering" Computer Graphics Forum, 20, 3, Sept. 2001.

[9] S. E Humphris, R.A. Zierenberg, L.S. Mullineaux, and R.E. Thomson, eds., Seafloor Hydrothermal Systems: Physical, Chemical, Biological and Geological Interactions, Geophysical Monograph 91, American Geophysical Union, Washington, D.C., 1995

[10] R. A. Feely, M. Lewison, G. J. Massoth, G. Robert-Baldo, J. W. Lavelle, R. H. Byrne, K. L. Von Damm, and H. C. Curl, Jr., "Composition and dissolution of black smoker particulates from active vents on the Juan de Fuca Ridge”, Journal of Geophysical Research, 92 (B11), 11347-11363, 1987.

[11] N. Max, "Optimal models for direct volume rendering", IEEE Transactions on Visualization and Computer Graphics, 1(2), 99108, 1995

[12] H. Medwin and C. S. Clay, "Chapter 7: Sound scattered by a body", Fundementals of Acoustical Oceanography, Academic Press, Boston, 1997.

[13] J. T. Kajiya and B. P. Von Herzen, "Ray tracing volume densities, Computer Graphcs, 18, 3 (July), 1984, pp. 165-174.

[14] J. Kniss, S. Premoze, C. Hansen, P. Shirley, and A. McPherson, “A Model for Volume Lighting and Modeling", IEEE Transactions on Visualization and Computer Graphics, 9,2 (April-June), 2003, pp. 150-162.

[15] T. Nishita, Y. Dobashi, and E. Nakamae, "Display of Clouds Taking into Account Multiple Anisotropic Scattering and Sky Light”, Proceedings of the 23rd Annual Conference on Computer Graphics and Interactive Techniques, 1996, pp. 379-386.

[16] D. R. Palmer, "Rayleigh scattering from nonspherical particles", $J$. Acoust. Soc. Am., 99, 1996, pp. 1901-1912.

[17] D. R. Palmer, P. A. Rona, and M. J. Mottl, "The acoustics of 'black smoker' hydrothermal plumes”, J. Acoust. Soc. Am, 80, 1986, pp. 888-898.

[18] R. Pope and E. Fry, "Absorption spectrum (380-700nm) of pure water. II. Integrating cavity measurements”, Applied Optics, 36 (33), 1997, pp. 8710-8723.

[19] K. Riley, D. Ebert, C Hansen, and J. Levit, "Visually Accurate Multi-Field Weather Visualization”, IEEE Visualization 2003 Proceedings, 2003, pp. 279-292.

[20] P. A. Rona, K.G. Bemis, D. Kenchammana-Hosekote, and D. Silver, "Acoustic imaging and visualization of plumes discharging from black smoker vents on the deep seafloor”, IEEE Visualization 1998 Proceedings, 1998, pp. 475-478.

[21] P. A. Rona, D. R. Jackson, K. G. Bemis, C. D. Jones, K. Mitsuzawa, D. R. Palmer, and D. Silver, "Acoustics advances study of sea floor hydrothermal flow”, EOS, Trans. AGU, 83(44), October 29, 2002, pp. $497 \& 501-502$.

[22] P. A. Rona, D. R. Jackson, K. G. Bemis, C. D. Jones, K. Mitsuzawa, D. R. Palmer, and D. Silver, "A new dimension in investigation of seafloor hydrothermal flows", RIDGE 2000 Events, 1(1), 2003, pp. 26-29.

[23] M. Rudnicki and H. Elderfield,. "A chemical model of the buoyant and neutrally buoyant plume above the TAG vent field, 26 degrees N, Mid-Atlantic Ridge", Geochimica et Cosmochimica Acta, 57 (13), 1993, pp. 2939-2957.

[24] K. Santilli, "Realistic Images from Hydrothermal Plume Data", http://www.caip.rutgers.edu/ kls93/realplume

[25] J. Schpok, J. Simons, D. S. Ebert, C. Hansen, "A Real-Time Cloud Modeling, Rendering, and Animation System", Proceedings of the 2003 ACM SIGGRAPH/Eurographics Symposium on Computer Animation, 2003, pp. 160-166.

[26] Stephen Low Productions, Inc., Volcanoes of the Deep Sea, 2003, http://www.volcanoesofthedeepsea.com.

[27] S. L. White, A. D. Chave and G. T. Reynolds, "Investigations of ambient light emission at deep-sea hydrothermal vents", Journal of Geophysical Research, 107 (B1), 10.1029/2000JB000015, 2002. 

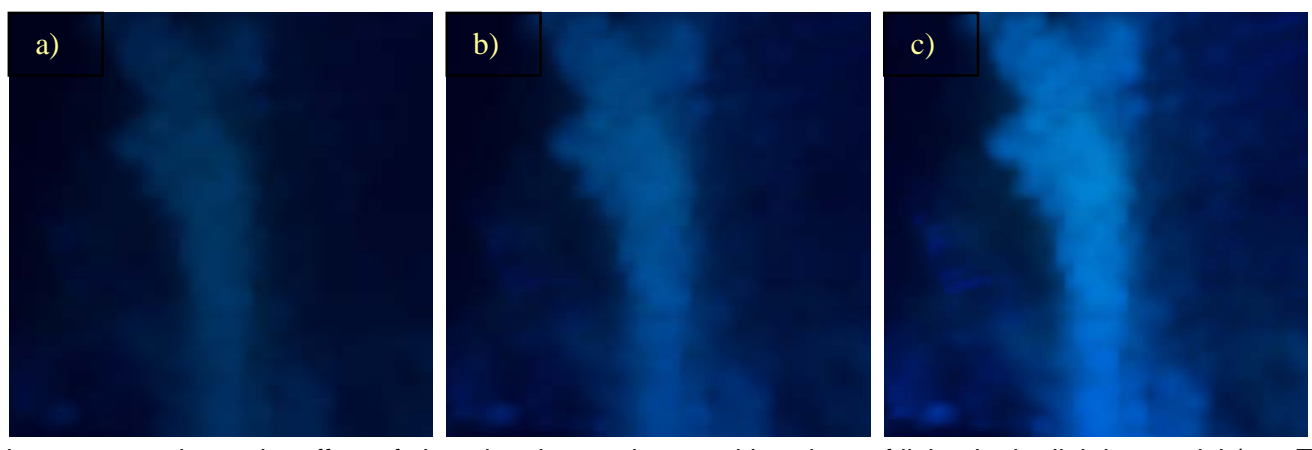

Figure 9. This sequence shows the effect of changing the numbers and locations of lights in the lighting model (see Table 4 for the exact positions). (a) The cruise lighting setup has 2 lights close to the camera. The plume is very hard to see consistent with the observation that during the acquisition of the acoustic data, it was not possible to see the plume at all. (b) The IMAX 3 lighting set up has 3 lights, two of which are farther from the camera. The plume is brighter with more light and some internal shadows are visible. (c) The IMAX 5 lighting setup has 5 lights and hence the plume is much more visible. Internal shadows make the eddies very visible in this image.
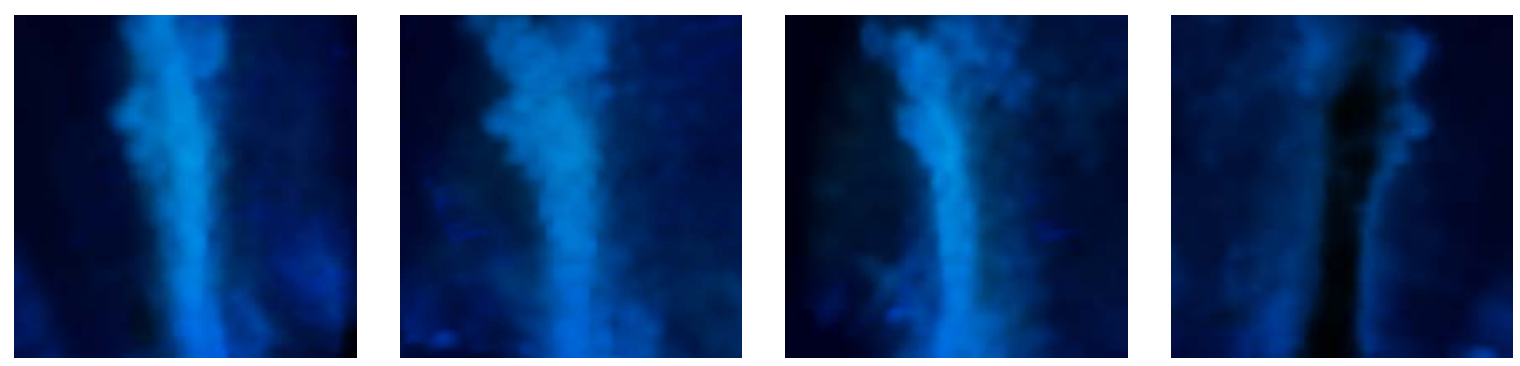

Figure 10. Lighting panorama of the acoustic data using the IMAX 5 setup. Viewing angles from left to right are $-45^{\circ}, 0^{\circ}, 45^{\circ}$ and $180^{\circ}$. The $180^{\circ}$ view shows the glow of the lights around the edges of the opaque plume.
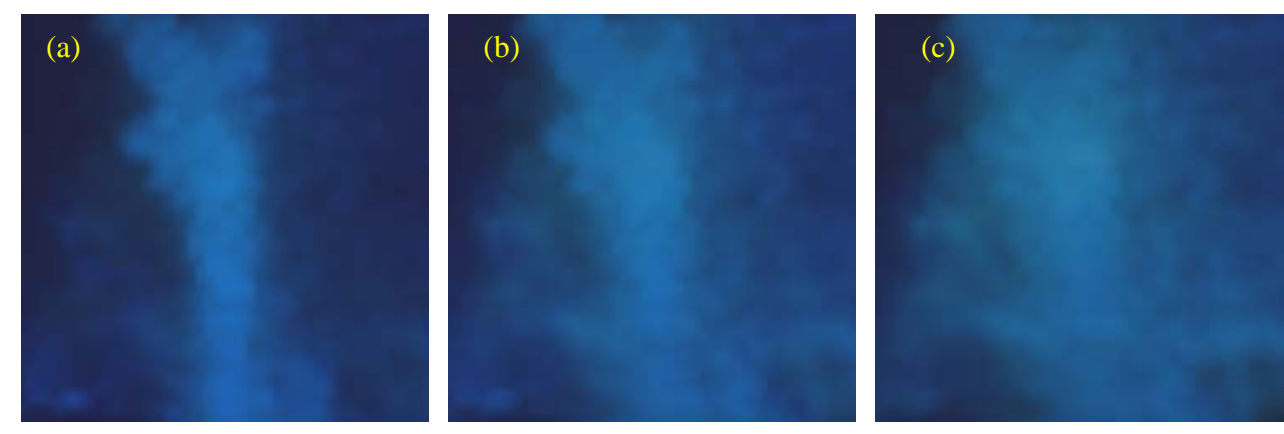

Figure 11. This sequence shows the variation in the generated images with the estimated extinction scale factor. This sequence uses the IMAX 3 lighting setup. (a) $\lambda=1.79 \times 104(1000 \mu \mathrm{m})$. (b) $\lambda=5.46 \times 104(800 \mu \mathrm{m})$. (c) $\lambda=1.00 \times 105(700 \mu \mathrm{m})$.
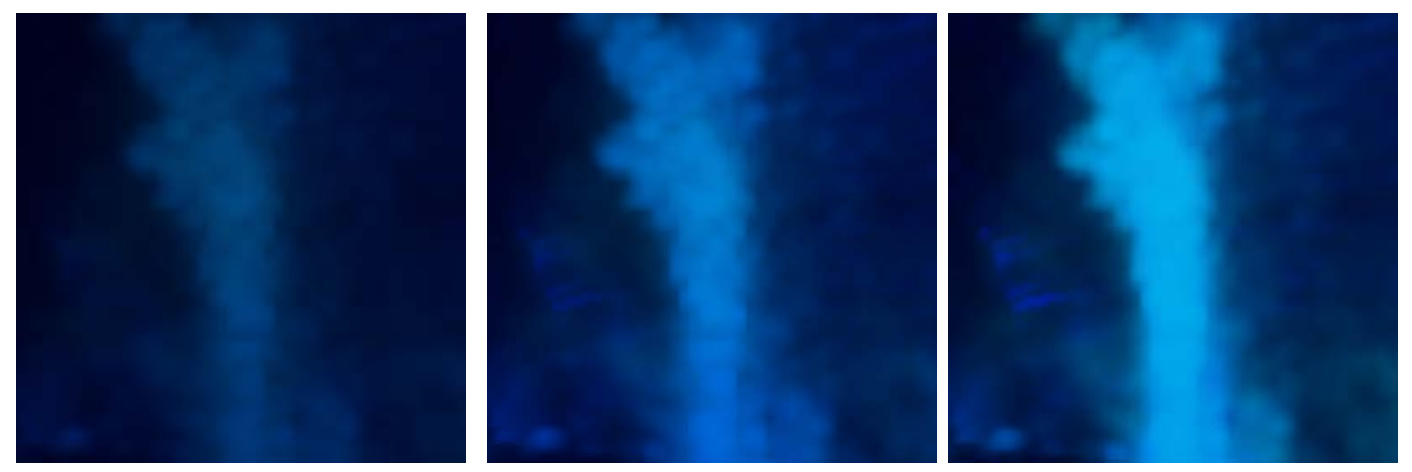

Figure 12. This sequence shows the variation in the generated images with the assumed albedo based on the chemical composition of the particles. The albedo varies left to right as 0.3 (chalcopyrite dominates), 0.5 (pyrite dominates), and 0.7 . 